\title{
3. Integrality of Eisenstein Liftings
}

\author{
By Shin-ichiro Mizumoтo \\ Department of Mathematics, Tokyo Institute of Technology \\ (Communicated by Shokichi IyANAGA, M. J. A., Jan. 14, 1991)
}

Introduction. In this note we report an integrality result on Fourier coefficients of Eisenstein series for Siegel modular groups attached to cusp forms. Our result, specialized to the case where the cusp form is a constant, coincides with the theorem of Siegel [9] and Böcherer [1] on Siegel's Eisenstein series. Details will appear elsewhere.

1. Eisenstein lifts of cusp forms. For $n, k \in Z_{>0}$, let $M_{k}^{n}$ (resp. $S_{k}^{n}$ ) be the $C$-vector space of holomorphic Siegel modular (resp. cusp) forms of weight $k$ with respect to $\Gamma_{n}:=\operatorname{Sp}(n, \boldsymbol{Z})=\mathrm{Sp}_{2 n}(\boldsymbol{Z})$. For $r \in \boldsymbol{Z}$ such that $0 \leq r \leq n$, let $\Delta_{n, r}$ be the subgroup of $\Gamma_{n}$ consisting of all $M \in \Gamma_{n}$ whose entries in the first $n+r$ columns and last $n-r$ rows vanish. For $f \in S_{k}^{r}$ with $k$ even $>0$, the Eisenstein series of Langlands-Klingen type [5, 3] is defined by:

$$
[f]_{r}^{n}(Z, s)=\sum_{M: \Delta_{n, r} \Gamma_{n}}\left(\frac{\operatorname{det}(\operatorname{Im}(M\langle Z\rangle))}{\operatorname{det}\left(\operatorname{Im}\left(M\langle Z\rangle^{*}\right)\right)}\right)^{s} f\left(M\langle Z\rangle^{*}\right) j(M, Z)^{-k} .
$$

Here $s \in C$ with $\operatorname{Re}(s)>(n+r+1-k) / 2 ; Z$ is a variable on the Siegel upper half space of degree $n$; for $M=\left(\begin{array}{ll}A & B \\ C & D\end{array}\right)$ with $n \times n$ matrices $A, B, C, D$, we put $M\langle Z\rangle=(A Z+B)(C Z+D)^{-1}$ and $j(M, Z)=\operatorname{det}(C Z+D) ; M\langle Z\rangle^{*}$ is the upper left $r \times r$ block of $M\langle Z\rangle$. We understand that $S_{k}^{0}=C$. The righthand side of (1) converges absolutely and locally uniformly for $(Z, s)$ in the above region; moreover $[f]_{r}^{n}(Z, s)$ as a function in $s$ has a meromorphic continuation to the whole $s$-plane.

Suppose $f$ is an eigenform, i.e. a non-zero eigenfunction of the Hecke algebra. Let $L(s, f, \mathrm{St})$ be the standard $L$-function attached to $f$ in the sense of [2]. We understand that each $f \in S_{k}^{0}-\{0\}$ is an eigenform with $L(s, f, \underline{\mathrm{St}}):=\zeta(s)$. We assume that either of the following two conditions is satisfied:

$$
\begin{aligned}
& \text { (a) } k \equiv 0(\bmod 2) \text { and } k>(n+r+3) / 2 \text {, or } \\
& \text { (b) } k \equiv 0(\bmod 4) \text { and } k \geq r \text {. }
\end{aligned}
$$

Then [7], $L(s, f, \underline{\mathrm{St}})$ is holomorphic at $s=k-r$. Suppose

$$
\begin{cases}L(k-r, f, \mathrm{St}) \neq 0 & \text { if } k>r, \\ L^{\prime}(0, f, \mathrm{St}) \neq 0 & \text { if } k=r .\end{cases}
$$

Then $[7,10],[f]_{r}^{n}(Z, s)$ is holomorphic in $s$ at $s=0$, and $[f]_{r}^{n}(Z):=[f]_{r}^{n}(Z, 0)$ is an eigenform belonging to $M_{k}^{n}$.

Remark. The condition (3) is automatically satisfied if $k \geq 2 r+2$.

Let $\Phi$ be the Siegel operator. We have $\Phi^{n-r}[f]_{r}^{n}=2^{c} f$ with $c=0$ or 1 ; 
$c=1$ if and only if $k \equiv 0(\bmod 4)$ and $r+1 \leq k \leq(n+r+1) / 2$.

We write the Fourier expansion of $[f]_{r}^{n}$ as

$$
[f]_{r}^{n}(Z)=\sum_{T \geq 0} a\left(T,[f]_{r}^{n}\right) \exp (2 \pi i \operatorname{trace}(T Z))
$$

Here $T$ runs over all symmetric positive semi-definite semi-integral matrices of size $n$.

Let $V_{0}^{(r)}$ be the $C$-subspace of $S_{k}^{r}$ spanned by the eigenforms satisfying the condition (3). Then [ $]_{r}^{n}$ defines a linear map from $V_{0}^{(r)}$ to $M_{k}^{n}$. For $F \in\left[V_{0}^{(s)}\right]_{s}^{r}$ with $0 \leq s \leq r$, we put $[F]_{r}^{n}:=\left[\Phi^{r-s} F\right]_{s}^{n}$.

2. Statement of result. Let $f \in S_{k}^{r}$ be an eigenform with $r \in Z_{\geq 0}$. For $r=0$, we understand that the Hecke operators are the scalar multiplications and that the Petersson inner product is defined by $(a, b):=a \bar{b}$ for $a, b \in S_{k}^{0}=C$. Let $\boldsymbol{Q}(f)$ be the totally real number field [4] generated by the eigenvalues of the Hecke operators over $\boldsymbol{Q}$ on $f$ and let $Z(f)$ be the ring of integers in $\boldsymbol{Q}(f)$. In particular, $\boldsymbol{Q}(f)=\boldsymbol{Q}$ if $f \in S_{k}^{0}$.

Remark. There exists a basis $\left\{f_{j}\right\}$ for $S_{k}^{r}$ with each $f_{j}$ being an eigenform whose Fourier coefficients belong to $\boldsymbol{Q}\left(f_{j}\right)$.

For a subring $R$ of $C$, let $T_{R}$ be the algebra of Hecke operators over $R$ acting on $M_{k}^{r}$ (cf. [4]). For a $C$-subspace $W$ of $M_{k}^{r}$, we denote by $W(R)$ the $R$-module consisting of the elements in $W$ whose Fourier coefficients belong to $R$.

Lemma. Let $f \in S_{k}^{r}$ be an eigenform satisfying (2) and (3). Suppose that the Fourier coefficients of $f$ belong to $\boldsymbol{Q}(f)$. Then there exist a $\boldsymbol{T}_{Q^{-}}$ simple component $V$ of $V_{0}^{(r)}(\boldsymbol{Q})$ and a $\boldsymbol{T}_{\boldsymbol{Q}}$-submodule $V^{\prime}$ of $V_{0}^{(r)}(\boldsymbol{Q})$ such that: $f \in V \otimes_{Q} C$ and $V^{\prime} \otimes_{Q} C=\left(V \otimes_{Q} C\right)^{\perp}$; here $\perp$ denotes the orthogonal complement in $V_{0}^{(r)}$ with respect to the Petersson inner product.

Let $f$ be as in the lemma. Let $T f=\lambda(T, f) f$ for $T \in T_{C}$. We denote by $\kappa(f)$ the exponent (as a finite abelian group) of $Z(f) / Z\left[\lambda(T, f) \mid T \in T_{Z}\right]$, and by $\nu(f)$ the exponent of $V_{0}^{(r)}(Z) /\left(V(Z) \oplus V^{\prime}(Z)\right)$. Let $\mathfrak{D}(\boldsymbol{Q}(f))$ be the different of $\boldsymbol{Q}(f) / \boldsymbol{Q}$. We define $\mathfrak{Y}(f):=\kappa(f) \nu(f) \mathfrak{D}(\boldsymbol{Q}(f))$.

If $r \in Z_{>0}$, we put $W_{0}:=\oplus_{j=0}^{r-1}\left[V_{0}^{(j)}\right]_{j}^{r-1}$. Noting that each [ $]_{j}^{r-1}$ preserves the rationality of Fourier coefficients, we denote by $\mu_{k}(r)$ the exponent of $\left[W_{0}(Z)\right]_{r-1}^{r} /\left[W_{0}\right]_{r-1}^{r}(Z)$. If $r=0$, we put $\mu_{k}(0):=1$.

Theorem. Let $k, n \in Z_{>0}$. Let $f \in S_{k}^{r}$ be an eigenform with $0 \leq r \leq n$. Suppose that $k \geq \max (r+1, n / 2)$ and that the conditions (2) and (3) are satisfied. Assume that the Fourier coefficients of $f$ belong to $\boldsymbol{Q}(f)$ and that a Fourier coefficient of $f$ is equal to 1 . Then for each $T^{(n)}>0$ we have:

$$
\begin{gathered}
a\left(T,[f]_{r}^{n}\right) \in \mu_{k}(r)^{-1} \xi_{k, r}^{-1} L^{*}(k-r, f, \underline{\mathrm{St}})^{-1} \cdot 2^{n-\delta((n-2) / 4)} \\
\cdot \prod_{i=1}^{[n / 2]-\delta_{k, n / 2}} d_{i} \cdot \prod_{j=1}^{r} d_{j}^{-1} \cdot \prod_{l=r+1}^{\min (k-1,[(n+r) / 2])} n_{l}^{-1} \\
\cdot N_{k, n}^{-1} \mathfrak{T}(f)^{-1} .
\end{gathered}
$$

Here $\delta(x)=1$ or 0 according as $x \in Z$ or not; 


$$
\begin{gathered}
\xi_{k, r}:=2^{(r(r+5) / 2)-r k+2-k-\delta_{r, 0}} \pi^{-r / 2} \Gamma\left(k-\frac{r}{2}\right) \prod_{j=1}^{r} \Gamma\left(k-\frac{r+j}{2}\right)^{2} \in \boldsymbol{Q}_{>0} ; \\
L^{*}(k-r, f, \underline{\mathrm{St}}):=L(k-r, f, \underline{\mathrm{St}}) \pi^{-(2 r+1) k+3 r(r+1) / 2}(f, f)^{-1} \in \boldsymbol{Q}(f) ;
\end{gathered}
$$

the reduced denominator and numerator of the absolute value of $B_{2 k-2 i} /(k-i)=-2 \zeta(1-2 k+2 i)$ is denoted by $d_{i}$ and $n_{i}$ respectively; $N_{k, n}:=$ $\prod_{p \in \mathscr{P}} p[k-n / 2]_{p}$ with [ $]_{p}$ denoting the $p$-part if $k>n / 2$, where $\mathcal{Q}:=$ $\left\{p \in Z_{>0} \mid p:\right.$ prime, $(p-1) \mid(2 k-n), p:$ odd if $\left.n \neq 0(\bmod 4)\right\}$, and $N_{n / 2, n}:=1$.

Remarks. ( i ) If $k \equiv 0(\bmod 4)$ and $k<n / 2$, then $a\left(T,[f]_{r}^{n}\right)=0$ for all $T^{(n)}>0$.

(ii) The above theorem for $r=0$ coincides with the well-known result [1, 9] for Siegel's Eisenstein series.

(iii) Similar result holds also for $k=r>0$; in this case, $L^{\prime}(0, f, \underline{\mathrm{St}})$ appears instead of $L(k-r, f$, St $)$.

Example. Let $\Delta \in S_{12}^{1}$ be the normalized cusp form. Then for all $T^{(9)}>0$ we have:

$$
a\left(T,[\Delta]_{1}^{9}\right) \in \frac{2^{6} \cdot 3 \cdot 5 \cdot 11 \cdot 17 \cdot 19 \cdot 23}{7 \cdot 283 \cdot 617 \cdot 3617 \cdot 43867} Z .
$$

Thus the theorem contains some congruence properties of Fourier coefficients of $[f]_{r}^{n}$.

\section{References}

[1] S. Böcherer: Über die Fourierkoeffizienten der Siegelschen Eisensteinreihen. Manuscripta Math., 45, 273-288 (1984).

[2] ——: Ưber die Funktionalgleichung automorpher $L$-Funktionen zur Siegelschen Modulgruppe. J. reine angew. Math., 362, 146-168 (1985).

[ 3 ] H. Klingen: Zum Darstellungssatz für Siegelsche Modulformen. Math. Z., 102, 30-43 (1967).

[4] N. Kurokawa: On Siegel eigenforms. Proc. Japan Acad., 57A, 47-50 (1981).

[5] R. P. Langlands: On the Functional Equations Satisfied by Eisenstein Series. Lect. Notes in Math., 544, Springer (1976).

[6] S. Mizumoto: On integrality of certain algebraic numbers associated with modular forms. Math. Ann., 265, 119-135 (1983).

[7] — : Poles and residues of standard $L$-functions attached to Siegel modular forms (1989) (preprint).

[8] — - Integrality of critical values of triple product $L$-functions. Lect. Notes in Math., 1434, Springer, 188-195 (1990).

[9] C. L. Siegel: Über die Fourierschen Koeffizienten der Eisensteinschen Reihen. Mat. Fys. Medd. Danske Vid. Selsk., 34, nr. 6 (1964).

[10] R. Weissauer: Stabile Modulformen und Eisensteinreihen. Lect. Notes in Math., 1219, Springer (1986). 